\title{
Justiz und Verfahren im Spannungsfeld von gelehrter Literatur, gerichtlicher Praxis und bildlicher Symbolik
}

\section{Symposion zum 80. Geburtstag von Wolfgang Sellert}

am 06. November 2015 in Göttingen

Wolfgang Sellert war von 1977 bis zu seiner Emeritierung 2002 Professor für Deutsche Rechtsgeschichte und Bürgerliches Recht in Göttingen. Seit 1978 ist er Ordentliches Mitglied der dortigen Akademie. In vielen Ämtern und Funktionsstellen im In- und Ausland, unter anderem etwa als Dekan der juristischen Fakultät, Mitglied und Vorsitzender von Akademiekommissionen oder als Direktor des Deutsch-Chinesischen Instituts für Wirtschaftsrecht in Nanjing (VR-China), hat er entscheidende Weichen gestellt und Bleibendes geschaffen. Und nicht zuletzt hat er als Person und Wissenschaftler seit nunmehr über einem halben Jahrhundert insbesondere die rechtsgeschichtliche Forschung, nicht nur zur Vormoderne, ganz wesentlich vorangetrieben und in Deutschland und weit darüber hinaus durch Wort und Tat maßgeblich geprägt: Mehr als Grund genug für die Göttinger Universität und Akademie der Wissenschaften, einen ihrer herausragenden Vertreter nicht nur mit einer feierlichen Abendveranstaltung, die zahlreiche Kollegen, Freunde und Wegbegleiter des Jubilars aus aller Welt in Göttingen zusammenführte, sondern auch mit einem von seiner Lehrstuhlnachfolgerin Eva Schumann glänzend organisierten wissenschaftlichen Symposion zu ehren.

Den Auftakt zu diesem gelehrten Teil der Feierlichkeiten bildete der fulminante Abendvortrag von Heiner Lück (Halle), ,,Daß ich erkenne, was die Welt im Innersten zusammenhält...' Gericht und Verfahren als Zentralkategorien im Werk von Wolfgang Sellert“. Lück gelang es, aus großem eigenen Fachwissen einem in weiten Teilen fachfremden Publikum vormoderne Gerichte und Verfahren in einem übergreifenden Sinn als Orte und Medien von Information, Kommunikation und Integration, deren Verklammerung mit der Welt schlechthin also, in ungemein anschaulicher und lebendiger Weise begreiflich zu machen. Sein programmatischer Aufriss wirkte zugleich als überzeugendes Plädoyer für die integrative, im Sinne von Fachgrenzen überwindende und unterschiedliche Perspektiven verbindende Kraft der Rechtsgeschichte, der Person und Wirken Wolfgang Sellerts gleichermaßen verpflichtet sind. Dessen rechtsgeschichtliche Forschungen kreisen seit seiner Dissertation von 1964 und vor allem seit der 1973 erschienenen Habilitationsschrift um die höchste Gerichtsbarkeit im Alten Reich, insbesondere um den kaiserlichen Reichshofrat, der bis dahin im Schatten des vermeintlich moderneren Reichskammergerichts gestanden hatte. Es gehört zu den vielen bleibenden Verdiensten Sellerts, diese schiefe Perspektive durch unermüdliches Forschen und Publizieren allmählich zurechtgerückt zu haben. Dies 
hat den modernen historischen Wissenschaften jedweder Couleur den Weg gewiesen für die Erforschung des Reichshofrats als eine der zentralen Institutionen des Alten Reichs mit enormer Wirkkraft für die imperiale Symbiose von Herrschaft und Justiz wie auch für die Verklammerung von Reich, Territorien und Regionen und der auf diesen Ebenen auch und gerade im Lichte des reichshofrätlichen Wirkens erkennbaren rechtlichen, sozialen und ökonomischen Interaktionen. Seine Habilitationsschrift über das reichshofrätliche Verfahren trotzt seit beinahe einem halben Jahrhundert allen wissenschaftlichen „Turns“ und bildet selbst für hochaktuelle fächerübergreifende Forschungsdiskurse wie etwa über „Justiznutzung“ oder „Infrajustiz“, von denen seiner Zeit noch niemand etwas ahnen konnte, unverzichtbares Orientierungswissen.

Solches Erkenntnisstreben mag bisweilen faustische Dimensionen annehmen. Mindestens ebenso entscheidend sind aber die eher irdischen Belange seiner Umsetzung, die faustische Tat, wenn man so will. Diesen Weg vom Willen zur Tat schilderte der ehemalige Wiener Archivdirektor Leopold Auer (Wien) in seinem Kurzvortrag über „Wolfgang Sellert und das Projekt zur Erschließung der Prozessakten des Reichshofrats“. Spätestens nachdem Sellerts langjähriger Weggefährte Bernhard Diestelkamp in den 1970er Jahren die heute weitgehend abgeschlossene Verzeichnung der Reichskammergerichtsakten angestoßen hatte, und die Forschung deren Ergebnisse zunehmend aufgriff, war klar, dass die angesprochene Schieflage in der Bewertung der Reichsjustiz und damit auch des Alten Reichs insgesamt sich unweigerlich verstetigen würde, wenn nicht auch die im Wiener Haus-, Hof- und Staatsarchiv lagernden und mit ca. 70.000 Stücken ähnlich umfangreichen Judizialakten des Reichshofrats erschlossen würden. Nach zahllosen Korrespondenzen, Gesprächen und Sitzungen seit 1992 konkretisierte sich die Idee eines solchen Unternehmens 1999 zunächst in Form eines von der VolkswagenStiftung getragenen, von Eva Ortlieb bearbeiteten und von Auer mit großer Expertise vor Ort unterstützten Pilotprojekts. 2002 fand das dadurch methodisch erprobte Vorhaben Aufnahme in das Göttinger Akademienprogramm. 2006 gelang der erhoffte Durchbruch, als die Union der deutschen Akademien das Vorhaben als deutsch-österreichisches Kooperationsprojekt unter Federführung der Göttinger Akademie mit einer Laufzeit bis vorerst 2024 bewilligte. 2007 nahm die Wiener Arbeitsstelle, die einzige der Göttinger Akademie, die ihren Sitz im Ausland hat, mit zwei wissenschaftlichen Mitarbeitern sowie einer Hilfskraft unter der Leitung von Wolfgang Sellert ihre Arbeit an der Verzeichnung des größten zusammenhängenden Aktenbestandes auf, den das Alte Reich hinterlassen hat.

Dass auch mit einem solch vergleichsweise kleinen Projektteam zusammen mit einem engagierten Projektleiter, der mit der ihm eigenen Akribie und Sorgfalt die Verzeichnungsarbeit begleitet, und trotz der besonderen logistischen, organi- 
satorischen und nicht zuletzt auch persönlichen Herausforderungen, die ein im Ausland durchgeführtes Projekt an alle Beteiligten stellt, beachtliche Ergebnisse erzielt werden können, zeigte der eindrückliche Kurzbericht von Ortliebs Nachfolger Tobias Schenk (Wien), „Die Erschließung der Reichshofratsakten. Eine Zwischenbilanz nach acht Jahren“: Bislang sind unter Sellerts Herausgeberschaft in sieben stattlichen Bänden mit insgesamt 5174 Druckseiten die Verzeichnungen von 6207 Akten des 16. und 17. Jahrhunderts veröffentlicht worden. Zwei weitere Bände mit rund 2300 Aktenverzeichnungen stehen unmittelbar vor dem Abschluss bzw. vor der Drucklegung. Die parallele Publikation im Archivinformationssystem des österreichischen Staatsarchivs ist inzwischen beschlossen. Die weltweite Verfügbarkeit der Verzeichnungsergebnisse nach dem Open-AccessPrinzip in einem verlässlichen modernen Kommunikationsmedium wird dadurch auf Dauer gewährleistet. Insgesamt sind bereits Akten im Gesamtumfang von deutlich über einer halben Million Blätter (ca. 1 Million Seiten) nach wissenschaftlichen Kriterien verzeichnet worden, die sich im Zuge des kollektiven Arbeitsprozesses immer weiter verfeinert haben und nun die Grundlage für die Vision einer über die Projektdauer hinausgehenden Verzeichnung des Gesamtbestandes bilden. Schenk wies in seinem illustrativen Werkstattbericht sehr zu Recht darauf hin, dass Erschließung mehr bedeutet als Verzeichnung, indem im Zuge der Verzeichnung jedes einzelne dieser Blätter foliiert, jede Akte in neue säurefreie Mappen verpackt, beschriftet und in ebenfalls aktuellen archivischen Konservierungsstandards genügenden Kartons gelegt wird. Das Projekt (http://www.reichshofratsakten.de) leistet damit in enger Kooperation mit dem Archiv nicht nur durch Wort, sondern auch durch Tat einen unumgänglich notwendigen und bleibenden Beitrag zur Bewahrung und dauerhaften wissenschaftlichen Nutzung eines einzigartigen europäischen Kulturguts.

Schon bevor die Idee zu diesem derzeit größten wissenschaftlichen Aktenerschließungsprojekt in der deutschen Forschungslandschaft konkrete Gestalt annahm, hatte Wolfgang Sellert mit seiner zweibändigen Edition der Ordnungen des Reichshofrats die zentralen normativen Quellen zu Verfassung und Verfahren dieses höchsten Gerichts herausgegeben (1980/1990). In Ergänzung dazu sprach Sellerts Schüler Peter Oestmann (Münster) in seinem Vortrag „Gemeine Bescheide des Reichshofrats zwischen Prozesspraxis und Gesetzgebung“ über Eigennormsetzung der Gerichte in Form von Einzelverordnungen. Diese fiel allerdings im Fall des Reichshofrats im Gegensatz zum Reichskammergericht vergleichsweise bescheiden aus und zielte hauptsächlich auf die Tätigkeit der Agenten und Prokuratoren, während die punktuelle Justierung der Gerichtsordnung am Reichshofrat wiederum im Gegensatz zum Reichskammergericht Gegenstand der nicht zu den Gemeinen Bescheiden („Decreta communia“) zu rechnenden kaiserlichen Dekrete („Decreta Caesarum“) war. Oestmann bereitet die den Reichshofrat be- 
treffende Fortsetzung seiner Edition der Gemeinen Bescheide der Höchstgerichte vor, aus der er vorab einige Beispiele vorstellte, und auf die man gespannt sein darf. Sein treffliches Wort vom Staub der Archive als Dünger der Rechtsgeschichte bewahrheitete sich einmal mehr durch den innovativen Vortrag von Anja AmendTraut (Würzburg), „Sich der Concurrenz erwehren...' Kaufmännischer Wettbewerb unter höchstgerichtlicher Kontrolle“. Amend-Traut nutzt seit langem und mit erstaunlichen Ergebnissen die enorme Bandbreite reichsgerichtlicher Akten zu Fragen der vormodernen Wirtschaftsgeschichte, in diesem Fall Akten zu reichskammergerichtlichen Verfahren im Streit der Hamburger Formschneider um Geheimhaltungsklauseln in Gesellschafts- und Dienstverträgen sowie insbesondere im Streit um Frankfurter Tabaksmarkenzeichen. Die Prozesse drehten sich im Kern um auch heute noch aktuelle Probleme der Qualitätssicherung von handwerklichen Produkten und des Markenschutzes. Ihre Untersuchung warf zudem aufschlussreiche Schlaglichter auf die Rolle des Privilegs, das ein vor höheren Instanzen einklagbares Recht schuf und damit vielleicht eine Art Zwischenschritt im Transformationsprozess vom genossenschaftlich-zünftischen zum allgemeinen patentrechtlichen Markenschutz im Wirtschaftsprivatrecht des 19. Jahrhunderts markierte.

Bis dahin war es aber noch ein weiter Weg, der mit einem Fundamentalvorgang der europäischen Rechtsgeschichte begann, nämlich mit der Rezeption des römischen Rechts im vormodernen Europa. Karin Nehlsen-von Stryks (Freiburg) Vortrag, „Zur Typologie spätmittelalterlicher Zivilverfahren: Tradition und gelehrtes Recht“, widmete sich dem Tagungsthema entsprechend der prozessgeschichtlichen Seite dieses Vorgangs. Inwieweit haben sich in dessen spätmittelalterlicher Frühphase die parallel praktizierten traditionellen und römisch-rechtlichen Arten des Zivilprozesses gegenseitig beeinflusst? Aufgrund eines kundigen Vergleichs zentraler Prozesselemente wie etwa der Stellung der Parteien, der Beweisverfahren und der Urteilsfindung kam sie zu dem Schluss, dass die Unterschiede zu relativieren sind und gelehrte Prozessformen die traditionellen mehr geprägt haben als umgekehrt. Bekanntlich führte die Rezeption in sozialgeschichtlicher Hinsicht zur Formierung und zum Aufstieg der juristischen Funktionseliten. Garant dieser Erfolgsgeschichte war laut Alain Wijffels (Louvain/Leuven), „Justiz und Gutes Regiment - Die Gemälde von Hans Vredeman de Vries für das Rathaus von Danzig (1593-1596)“, die durch Rechtsstudium erworbene Fähigkeit des gleichermaßen gerechten wie auch effektiven Gestaltens. Wijffels stellte interessante Bildzeugnisse öffentlicher Beschwörung des so definierten vormodernen „public gouvernance“ vor und diskutierte zentrale Bildformeln, wie etwa die Binde der Justitia, die Recht spricht ohne Ansehen der Parteien. Oder darf sie dort, wo sie sich eng mit dem Regiment verbindet, nicht immer so genau hinsehen? Jedenfalls scheint die Berücksichtigung konkreter Entstehungs- und Verwen- 
dungskontexte - wie im Falle von Texten - auch bei der Interpretation von Bildern die Erkenntnismöglichkeiten durchaus zu steigern. Ein wichtiges methodisches Instrument moderner Bildwissenschaft ist darüber hinaus der Bildvergleich, wie Andreas Deutsch (Heidelberg), „Inszenierte Macht? Bildliche Darstellung gerichtlicher Praxis - Von der höchsten Reichsgerichtsbarkeit bis zum Dorfgericht“, vorführte. Seine präzisen Bildanalysen ließen keinen Zweifel daran, dass die wenigen, meistens in der Publizistik des 18. Jahrhunderts existierenden Darstellungen der Höchstgerichte sehr stark voneinander abhängig sind, mithin der realgeschichtliche Quellenwert einzelner Bilder im Sinne von fotoähnlichen Zeugnissen überaus gering ist. Das Schweigen der Bildquellen zu den Höchstgerichten hat etwas mit dem allmählichen Ausschluss der Öffentlichkeit von den Verfahren zu tun, den die zunehmende Rezeption des gelehrten Rechts im Allgemeinen brachte. Vielleicht erklärt dieser Umstand auch den Generalbefund des Vortrags von Gernot Kocher, „Rechtswahrung auf höchster Ebene - Ein ikonographischer Exkurs vom Hochmittelalter bis zum Reichshofrat“, der seit langem in Graz Bilder sammelt, sichtet und ordnet sowie dort auch eine im Netz zugängliche „Rechtsikonographische Datenbank“ eingerichtet hat. Sein Vortrag lebte von der Flut faszinierender Bilder, die bezeichnender Weise mit dem Ende des 16. Jahrhunderts verebbte.

Insgesamt lotete das anregende Symposion fallweise die offenbar gerade im thematischen Umfeld von Gericht und Verfahren ungemein reichhaltigen Möglichkeiten neuer fachspezifischer, aber auch fächerübergreifender und anschlussfähiger Forschungen aus, die die Erschließung von Akten und die Entdeckung und Sammlung von Bildquellen bieten. Vermutlich ist die auch in anderen historisch orientierten Kulturwissenschaften $\mathrm{zu}$ beobachtende zunehmende Rückbesinnung auf diese Urquellen immer noch der beste aller „turns“.

Ulrich Rasche, Wien 